Pacific

Journal of

Mathematics

ZEROS OF EXTREMAL FUNCTIONS IN WEIGHTED BERGMAN SPACES

RACHEL J. WEIR 


\title{
ZEROS OF EXTREMAL FUNCTIONS IN WEIGHTED BERGMAN SPACES
}

\author{
RACHEL J. WEIR
}

\begin{abstract}
For $-1<\alpha \leq 0$ and $0<p<\infty$, the solutions of certain extremal problems are known to act as contractive zerodivisors in the weighted Bergman space $A_{\alpha}^{p}$. We show that for $0<\alpha \leq 1$ and $0<p<\infty$, the analogous extremal functions do not have any extra zeros in the unit disk and, hence, have the potential to act as zero-divisors. As a corollary, we find that certain families of hypergeometric functions either have no zeros in the unit disk or have no zeros in a half-plane.
\end{abstract}

\section{Introduction.}

For $-1<\alpha<\infty$ and $0<p<\infty$, the weighted Bergman space $A_{\alpha}^{p}$ is the space of functions $f$ analytic in the unit disk $\mathbb{D}$ for which

$$
\|f\|_{p, \alpha}^{p}=\int_{\mathbb{D}}|f(z)|^{p} w_{\alpha}(z) d \sigma(z)<\infty,
$$

where $w_{\alpha}(z)=(\alpha+1)\left(1-|z|^{2}\right)^{\alpha}$ and $d \sigma$ is normalized area measure on the disk. When $\alpha=0$, this space is precisely the unweighted Bergman space $A^{p}$. Hedenmalm [5] showed that contractive zero-divisors in $A^{2}$ can be constructed as solutions of extremal problems. This result was generalized to $A^{p}$ for $0<p<\infty$ by Duren, Khavinson, Shapiro and Sundberg [2, 3]. More precisely, given an $A^{p}$ zero-set $\left\{\zeta_{j}\right\}$ with $\zeta_{j} \neq 0$, the solution $G$ of the extremal problem

$$
\sup \left\{\operatorname{Re} f(0): f \in A^{p}, f\left(\left\{\zeta_{j}\right\}\right)=0,\|f\|_{p}=1\right\}
$$

is a contractive divisor. In other words, if $f \in A^{p}$ vanishes on $\left\{\zeta_{j}\right\}$, then $\|f / G\|_{p} \leq\|f\|_{p}$.

Shimorin $[\mathbf{9}, \mathbf{1 0}, \mathbf{1 1}]$ showed that analogous results hold in $A_{\alpha}^{2}$ for $-1<$ $\alpha \leq 1$ and in $A_{\alpha}^{p}$ for $-1<\alpha<0$ and $0<p<\infty$. On the other hand, it was shown by Hedenmalm and Zhu [7] that contractive divisors do not exist in $A_{\alpha}^{2}$ for $\alpha>1$. For $p \neq 2$, it is an open problem to determine the precise values of $\alpha$ for which the extremal functions will act as contractive divisors.

To act as a divisor, an extremal function must vanish only at the points in the prescribed zero-set. To examine when this is true, we first consider the case when the zero-set consists of a single point. Indeed, showing that the 
extremal function corresponding to a singleton zero-set has no extra zeros is a key step in proving the same for a general extremal function. For details see, for instance, [2].

In [13], we showed that for $-1<\alpha<\infty$ and $1 \leq p<\infty$ this single-point extremal function can be expressed in terms of a hypergeometric function. This representation is valid whenever it is known that the hypergeometric function is nonvanishing in the unit disk, in which case the extremal function will have no extra zeros in $\mathbb{D}$. Equivalently, if the extremal function is known a priori to have no extra zeros, the same technique can be used to establish the formula and, as a consequence, we may conclude that the corresponding hypergeometric function has no zeros in $\mathbb{D}$.

When $\alpha$ is a nonnegative integer, the hypergeometric functions in question are in fact polynomials. Using this fact and a result of Osipenko and Stessin [8], we were able to establish that the single-point extremal function in $A_{\alpha}^{p}$ has no extra zeros when $\alpha=1,2,3$ [13]. For other values of $\alpha$, describing the zeros of these hypergeometric functions directly is difficult.

In this paper, using techniques of Bergman space theory we establish a result about the zeros of hypergeometric functions. Indeed, we show that for $0<\alpha \leq 1$ and $0<p<\infty$ extremal functions in $A_{\alpha}^{p}$ have no extra zeros in the unit disk. In particular, the single-point extremal functions can not have any extra zeros in $\mathbb{D}$. From this we may conclude that the corresponding hypergeometric functions can not vanish in the unit disk.

The key step is to show that the single-point extremal functions in $A_{1}^{p}$ act as expansive multipliers on $A_{\alpha}^{p}$ for $0<\alpha \leq 1$, although they are constructed as functions in $A_{1}^{p}$. Since single-point extremal functions have no extra zeros when $\alpha=1$, the result follows. This method is a modification of the technique used by Shimorin [11] to prove that extremal functions in $A_{\alpha}^{p}$ have no extra zeros when $-1<\alpha<0$ and $0<p<\infty$. We also use properties of the weighted biharmonic Green function $\Gamma_{1}$ corresponding to the weight $w_{1}$. These properties can be established using the explicit formula for $\Gamma_{1}$ stated by Hedenmalm [6].

\section{Background.}

A sequence of points $\left\{\zeta_{j}\right\}$ is called an $A_{\alpha}^{p}$ zero-set if there is a function in $A_{\alpha}^{p}$ which vanishes precisely on this sequence. In particular, if a point $\alpha$ appears in the sequence $m$ times, then the function must have a zero at $\alpha$ of order exactly $m$. Given such a sequence $\left\{\zeta_{j}\right\}$ with $\zeta_{j} \neq 0$, we can pose the extremal problem

$$
\sup \left\{\operatorname{Re} f(0): f \in A_{\alpha}^{p}, f\left(\left\{\zeta_{j}\right\}\right)=0,\|f\|_{p, \alpha}=1\right\} .
$$

A normal families argument shows that for $\alpha>-1$ and $0<p<\infty$ an extremal function $G$ will exist. 
The Gauss hypergeometric function $F(a, b ; c ; z)$ is defined as

$$
F(a, b ; c ; z)=\sum_{n=0}^{\infty} \frac{(a)_{n}(b)_{n}}{(c)_{n}} \frac{z^{n}}{n !},
$$

where

$$
(x)_{n}=\frac{\Gamma(x+n)}{\Gamma(x)}=x(x+1) \ldots(x+n-1)
$$

is Pochhammer's symbol [1]. Here, $\Gamma$ denotes the usual Gamma function. In [13], we showed that for each $\beta \in \mathbb{D}$ the extremal function $G_{\beta}$ corresponding to the zero-set $\{\beta\}$ can be expressed in terms of hypergeometric functions. Indeed, for $-1<\alpha<\infty$ and $1 \leq p<\infty$,

$$
G_{\beta}(z)=\frac{\bar{\beta}}{|\beta|} \frac{\beta-z}{1-\bar{\beta} z}\left[\frac{F\left(-\alpha-1, \frac{p}{2} ; \frac{p}{2}+1 ; \bar{\beta} \frac{\beta-z}{1-\bar{\beta} z}\right)^{2}}{F\left(-\alpha-1, \frac{p}{2} ; \frac{p}{2}+1 ; 1\right) F\left(-\alpha-1, \frac{p}{2} ; \frac{p}{2}+1 ;|\beta|^{2}\right)}\right]^{\frac{1}{p}},
$$

provided that $F\left(-\alpha-1, \frac{p}{2} ; \frac{p}{2}+1 ; \zeta\right) \neq 0$ in the disk $\{|\zeta|<|\beta|\}$. In this case, $G_{\beta}$ will not vanish in $\mathbb{D}$ except for a simple zero at $\beta$. Conversely, if we assume a priori that $G_{\beta}$ vanishes only at $\beta$, we can derive the same formula and conclude that the function $F\left(-\alpha-1, \frac{p}{2} ; \frac{p}{2}+1 ; \zeta\right)$ is nonvanishing in $\{|\zeta|<|\beta|\}$.

For each fixed $\zeta$ in $\mathbb{D}$ the weighted biharmonic Green function $\Gamma_{\alpha}(z, \zeta)$ is the solution of the boundary-value problem

$$
\begin{cases}\Delta_{z}\left(\frac{1}{w_{\alpha}(z)} \Delta_{z} \Gamma_{\alpha}(z, \zeta)\right)=\delta_{\zeta}(z) & \text { for } z \in \mathbb{D} \\ \Gamma(z, \zeta)=\frac{\partial \Gamma_{\alpha}}{\partial n_{z}}(z, \zeta)=0 & \text { for } z \in \partial \mathbb{D} .\end{cases}
$$

Here, $\frac{\partial}{\partial n}$ is the outward normal derivative and $\Delta$ denotes one-fourth of the usual Laplacian. When $\alpha=0$, the function $\Gamma_{\alpha}$ is precisely the well-known biharmonic Green function $\Gamma$ which is given by the formula

$$
\Gamma(z, \zeta)=|z-\zeta|^{2} \log \left|\frac{z-\zeta}{1-\bar{\zeta} z}\right|^{2}+\left(1-|\zeta|^{2}\right)\left(1-|z|^{2}\right)
$$

For details of the derivation of this formula, see [4].

Essential to the proof of our main result is the fact that an explicit formula for the weighted biharmonic Green function is also known when $\alpha=1$. 
Indeed, Hedenmalm [6] presented the formula

$$
\begin{aligned}
\Gamma_{1}(z, \zeta)= & 2\left(|z-\zeta|^{2}-\frac{1}{4}\left|z^{2}-\zeta^{2}\right|^{2}\right) \log \left|\frac{z-\zeta}{1-\bar{\zeta} z}\right|^{2} \\
& +\frac{1}{4}\left(1-|z|^{2}\right)\left(1-|\zeta|^{2}\right)\left(7-|z|^{2}-|\zeta|^{2}-|z \zeta|^{2}-4 \operatorname{Re}(\bar{\zeta} z)\right) \\
& -\frac{1}{2}\left(1-|z|^{2}\right)^{2}\left(1-|\zeta|^{2}\right)^{2} \operatorname{Re}\left\{\frac{1+\bar{\zeta} z}{1-\bar{\zeta} z}\right\}
\end{aligned}
$$

The Green function $\Gamma_{1}$ is positive for $z, \zeta$ in $\mathbb{D}[\mathbf{6}]$. Moreover, the function $\Delta_{z} \Gamma_{1}(z, \zeta)$ has the form

$$
\Delta_{z} \Gamma_{1}(z, \zeta)=w_{1}(z)\left(\log \left|\frac{z-\zeta}{1-\bar{\zeta} z}\right|^{2}+H_{1}(z, \zeta)\right),
$$

where $H_{1}(\cdot, \zeta)$ is harmonic in $\mathbb{D}$ for fixed $\zeta \in \mathbb{D}$. Indeed, since

$$
\Delta\left(\log \left|\frac{z-\zeta}{1-\bar{\zeta} z}\right|^{2}\right)=\delta_{\zeta}(z)
$$

the function

$$
\frac{1}{w_{1}(z)} \Delta_{z} \Gamma_{1}(z, \zeta)-\log \left|\frac{z-\zeta}{1-\bar{\zeta} z}\right|^{2}
$$

must be harmonic in $\mathbb{D}$.

When $\alpha=1$ we are also able to establish some important properties of the single-point extremal functions. For the remainder of this paper, we will use the notation $G_{\beta}$ to denote the single-point extremal function in the space $A_{1}^{p}$. In other words, $G_{\beta}$ will be the solution of the extremal problem

$$
\sup \left\{\operatorname{Re} f(0): f \in A_{1}^{p}, f(\beta)=0,\|f\|_{p, 1}=1\right\} .
$$

By Theorem 4.1 in [13], the hypergeometric function

$$
F\left(-2, \frac{p}{2} ; \frac{p}{2}+1 ; z\right)=\frac{(p+2)(p+4)-2 p(p+4) z+p(p+2) z^{2}}{(p+2)(p+4)}
$$

does not vanish in $\mathbb{D}$ when $1 \leq p<\infty$. In fact, it is easy to see that it does not vanish in $\overline{\mathbb{D}}$. Hence, using Formula (2), we see that

$$
G_{\beta}(z)=\frac{\bar{\beta}}{|\beta|} \frac{\beta-z}{1-\bar{\beta} z}\left[\frac{P\left(\bar{\beta} \frac{\beta-z}{1-\bar{\beta} z}\right)^{2}}{8 P\left(|\beta|^{2}\right)}\right]^{\frac{1}{p}},
$$

where $P(z)=(p+2)(p+4)-2 p(p+4) z+p(p+2) z^{2}$. Consequently, $G_{\beta}(z) \neq 0$ in $\overline{\mathbb{D}}$ except for a simple zero at $\beta$. 
It is clear that $G_{\beta}(0)$ must be real and positive. Indeed, if not, multiplying $G_{\beta}$ by a suitable unimodular constant would produce a competitor function with larger real part. We can say more about $G_{\beta}(0)$.

Lemma 2.1. For $1 \leq p<\infty$, it is true that $0<G_{\beta}(0)<1$.

This result follows from the subharmonicity of $\left|G_{\beta}\right|^{p}$. The same arguments can be used to conclude that $0<G(0)<1$, where $G$ is an extremal function in $A_{\alpha}^{p}$ corresponding to an arbitrary $A_{\alpha}^{p}$ zero-set for $-1<\alpha<\infty$ and $0<p<\infty$.

\section{The expansive multiplier property.}

The first step in the proof of the main result is showing that $G_{\beta}$ acts as an expansive multiplier on $A_{\alpha}^{p}$ for $0<\alpha \leq 1$, where $G_{\beta}$ is the single-point extremal function in the space $A_{1}^{p}$. The technique used to establish this is a modification of a method of Shimorin [11]. From (6), it is clear that the function $G_{\beta}$ is analytic in $\overline{\mathbb{D}}$. Hence, the function $\left(\left|G_{\beta}(z)\right|^{p}-1\right) w_{1}(z)$ is continuous in $\overline{\mathbb{D}}$. Thus, the boundary value problem

$$
\begin{cases}\Delta \phi=\left(\left|G_{\beta}\right|^{p}-1\right) w_{1} & \text { in } \mathbb{D} \\ \phi=0 & \text { on } \partial \mathbb{D}\end{cases}
$$

has a unique solution $\phi$ in $C^{2}(\overline{\mathbb{D}})$.

Lemma 3.1. The solution $\phi$ has the following properties:

(i) $\frac{\partial \phi}{\partial n}=0$ on $\partial \mathbb{D}$

(ii) $\phi(\zeta)=\int_{\mathbb{D}} \Gamma_{1}(z, \zeta) \Delta\left(\left|G_{\beta}(z)\right|^{p}\right) d \sigma(z)$ for each $\zeta$ in $\mathbb{D}$.

Proof. (i) The proof is a modification of the proof of Lemma 3(a) in [2] and will be omitted. It uses the fact that

$$
\int_{\mathbb{D}}\left(\left|G_{\beta}\right|^{p}-1\right) f w_{1} d \sigma=0
$$

for all $f$ in $h^{\infty}$, which can be proved by adapting an argument given in [3].

(ii) We would like to show that, since $\phi=\frac{\partial \phi}{\partial n}=0$ on $\partial \mathbb{D}$, we can write

$$
\phi(\zeta)=\int_{\mathbb{D}} \Gamma_{1}(z, \zeta) \Delta\left(\frac{1}{w_{1}(z)} \Delta \phi(z)\right) d \sigma(z) .
$$

The result would then follow since $\Delta\left(\frac{1}{w_{1}} \Delta \phi\right)=\Delta\left(\left|G_{\beta}\right|^{p}-1\right)=\Delta\left(\left|G_{\beta}\right|^{p}\right)$. Identity (8) can be obtained as a consequence of Green's formula. However, since the integrand is smooth only in $\mathbb{D} \backslash\{\beta, \zeta\}$, we must apply Green's formula to the integral

$$
\int_{\mathbb{D}_{\epsilon}} \Gamma_{1}(z, \zeta) \Delta\left(\frac{1}{w_{1}(z)} \Delta \phi(z)\right) d \sigma(z)
$$


where $\mathbb{D}_{\epsilon}$ is the domain formed by removing from $\mathbb{D}$ the balls $B_{\zeta}=\{z$ : $|z-\zeta|<\epsilon\}$ and $B_{\beta}=\{z:|z-\beta|<\epsilon\}$. By letting $\epsilon \rightarrow 0$, the result follows from several estimates on the growth of $\phi$ and $\Gamma_{1}$. First, on $\partial B_{\beta}$,

$$
\left|\epsilon \frac{\partial}{\partial n}\left(\frac{1}{w_{1}} \Delta \phi\right)\right| \leq \epsilon p\left|G_{\beta}\right|^{p}\left|\frac{G_{\beta}^{\prime}}{G_{\beta}}\right|=O\left(\epsilon^{p}\right),
$$

as $\epsilon \rightarrow 0$.

Second, direct calculation using Formula (4) shows that

$$
\begin{aligned}
\epsilon \frac{1}{w_{1}\left(\zeta+\epsilon e^{i \theta}\right)} \Delta \Gamma_{1}\left(\zeta+\epsilon e^{i \theta}, \zeta\right) & =O(\epsilon \log \epsilon), \\
\left.\epsilon \frac{\partial}{\partial r} \Gamma_{1}\left(\zeta+r e^{i \theta}, \zeta\right)\right|_{r=\epsilon} & =O\left(\epsilon^{2} \log \epsilon\right),
\end{aligned}
$$

and

$$
\left.\epsilon \frac{\partial}{\partial r}\left(\frac{1}{w_{1}\left(\zeta+r e^{i \theta}\right)} \Delta \Gamma_{1}\left(\zeta+r e^{i \theta}, \zeta\right)\right)\right|_{r=\epsilon}=2+O(\epsilon)
$$

as $\epsilon \rightarrow 0$.

Using the properties of $\phi$ established in Lemma 3.1, we can now prove that $G_{\beta}$ is an expansive multiplier.

Theorem 3.2. For $0<\alpha \leq 1$ and $1 \leq p<\infty$, if $f \in A_{\alpha}^{p}$, then

$$
\left\|G_{\beta} f\right\|_{p, \alpha} \geq\|f\|_{p, \alpha} \text {. }
$$

Proof. Let $g=|f|^{p} h$, where $f$ is a polynomial and $h \in C^{2}(\overline{\mathbb{D}})$. Then

$$
\begin{aligned}
& \int_{\mathbb{D}}\left(\left|G_{\beta}(z)\right|^{p}-1\right) g(z) w_{1}(z) d \sigma(z) \\
& =\int_{\mathbb{D}} g(z) \Delta \phi(z) d \sigma(z) \\
& =\int_{\mathbb{D}} \phi(z) \Delta g(z) d \sigma(z) \\
& =\int_{\mathbb{D}} \int_{\mathbb{D}} \Gamma_{1}(z, \zeta) \Delta\left(\left|G_{\beta}(\zeta)\right|^{p}\right) \Delta g(z) d \sigma(\zeta) d \sigma(z),
\end{aligned}
$$

where the last step follows from Lemma 3.1 (ii). Although the derivatives of $g$ are not smooth near the zeros of the polynomial $f$, we can use arguments similar to those used to show (8) to justify our use of Green's formula.

For $n=0,1,2, \ldots$, we now let $g_{n}(z)=|f(z)|^{p} \sum_{k=0}^{n}(-1)^{k}\left(\begin{array}{c}\alpha-1 \\ k\end{array}\right)|z|^{2 k}$, where $f$ is a polynomial and $0<\alpha<1$. When $\alpha=1$, we let $g_{n}(z)=|f(z)|^{p}$. Each function $g_{n}$ is subharmonic. To see this, we need only observe that for each $k \in \mathbb{N}$ and for $p>0$, the function $\log \left(|f|^{p}|z|^{2 k}\right)$ is subharmonic and 
hence, by Jensen's inequality, the function $|f|^{p}|z|^{2 k}$ is subharmonic, since the exponential function is convex. As

$$
(-1)^{k}\left(\begin{array}{c}
\alpha-1 \\
k
\end{array}\right)=\frac{1}{k !}(1-\alpha)(2-\alpha) \ldots(k-\alpha)>0
$$

for $0<\alpha<1$, each function $g_{n}$ is a positive linear combination of subharmonic functions and must also be subharmonic. Setting $g=g_{n}$ in (9), we conclude that

$$
\int_{\mathbb{D}}\left(\left|G_{\beta}(z)\right|^{p}-1\right)|f(z)|^{p} \sum_{k=0}^{n}(-1)^{k}\left(\begin{array}{c}
\alpha-1 \\
k
\end{array}\right)|z|^{2 k} w_{1}(z) d \sigma(z) \geq 0,
$$

since $g_{n}$ is subharmonic and $\Gamma_{1}$ is positive. An application of the Dominated Convergence Theorem then shows that

$$
\int_{\mathbb{D}}\left(\left|G_{\beta}(z)\right|^{p}-1\right)|f(z)|^{p}\left(1-|z|^{2}\right)^{\alpha-1} w_{1}(z) d \sigma(z) \geq 0,
$$

from which we can conclude that

$$
\left\|G_{\beta} f\right\|_{p, \alpha} \geq\|f\|_{p, \alpha}
$$

for all polynomials $f$. Since $G_{\beta}$ is bounded and polynomials are dense in $A_{\alpha}^{p}$, the result follows.

\section{Boundary values of extremal functions.}

Before proving the main theorem, we first show that since $G_{\beta}$ is an expansive multiplier, its boundary values can not be too small. The ideas in this section generalize an approach of Dragan Vukotić [12].

The weighted Berezin transform $B_{\alpha} u$ of a function $u \in L^{1}\left(\mathbb{D}, w_{\alpha} d \sigma\right)$ is defined as

$$
\begin{aligned}
B_{\alpha} u(a) & =\int_{\mathbb{D}} u\left(\varphi_{a}(\zeta)\right) w_{\alpha}(\zeta) d \sigma(\zeta) \\
& =(\alpha+1) \int_{\mathbb{D}} \frac{\left(1-|a|^{2}\right)^{\alpha+2}\left(1-|z|^{2}\right)^{\alpha}}{|1-\bar{a} z|^{2 \alpha+4}} u(z) d \sigma(z),
\end{aligned}
$$

where $\varphi_{a}(\zeta)=\frac{a-\zeta}{1-\bar{a} \zeta}$.

Proposition 4.1. Let $a_{0} \in \partial \mathbb{D}$ and $u \in L^{1}\left(\mathbb{D}, w_{\alpha} d \sigma\right)$. If $\lim _{a \rightarrow a_{0}} u(a)=L$ exists and is finite, then $\lim _{a \rightarrow a_{0}} B_{\alpha} u(a)=L$.

Proof. Fix $\epsilon>0$. We can find $r>0$ for which $|u(z)-L|<\epsilon / 2$ whenever $z \in D_{0}=\mathbb{D} \cap D\left(a_{0}, r\right)$. It is not hard to show that $\left|\varphi_{a}^{\prime}(z)\right|^{\alpha+2} \rightarrow 0$ uniformly on compact subsets of $\overline{\mathbb{D}} \backslash\left\{a_{0}\right\}$ as $a \rightarrow a_{0}$. Hence, we can choose $a$ close enough to $a_{0}$ to ensure that

$$
\left|\varphi_{a}^{\prime}(z)\right|^{\alpha+2}<\frac{\epsilon}{2\left(\|u\|_{1, \alpha}+|L|\right)}
$$


for all $z \in \mathbb{D} \backslash D_{0}$. Noting that $\int_{\mathbb{D}}\left|\varphi_{a}^{\prime}(z)\right|^{2} w_{\alpha}\left(\varphi_{a}(z)\right) d \sigma(z)=1$, we see that

$$
\left|B_{\alpha} u(a)-L\right| \leq \frac{\epsilon}{2} \int_{D_{0}}\left|\varphi_{a}^{\prime}\right|^{2} w_{\alpha}\left(\varphi_{a}\right) d \sigma+\int_{\mathbb{D} \backslash D_{0}}|u-L|\left|\varphi_{a}^{\prime}\right|^{\alpha+2} w_{\alpha} d \sigma \leq \epsilon .
$$

Theorem 4.2. Suppose $-1<\alpha<\infty$ and $0<p<\infty$ and let $G$ be an expansive multiplier in $A_{\alpha}^{p}$. If $G$ has a continuous extension across the point $a_{0} \in \partial \mathbb{D}$, then $\left|G\left(a_{0}\right)\right| \geq 1$.

Proof. For $a \in \mathbb{D}$ let

$$
f_{a}(z)=\left(\frac{1-|a|^{2}}{(1-\bar{a} z)^{2}}\right)^{\frac{\alpha+2}{p}} .
$$

Then $f$ is in $A_{\alpha}^{p}$ since $\left\|f_{a}\right\|_{p, \alpha}=1$. Now by Proposition 4.1 , since $|G|^{p} \in$ $L^{1}\left(\mathbb{D}, w_{\alpha} d \sigma\right)$,

$$
\int_{\mathbb{D}}\left|f_{a} G\right|^{p} w_{\alpha} d \sigma=B_{\alpha}\left(|G|^{p}\right)(a) \rightarrow\left|G\left(a_{0}\right)\right|^{p}
$$

as $a \rightarrow a_{0}$. But the expansive multiplier property implies that for each $a \in \mathbb{D}$,

$$
\int_{\mathbb{D}}\left|f_{a} G\right|^{p} w_{\alpha} d \sigma \geq \int_{\mathbb{D}}\left|f_{a}\right|^{p} w_{\alpha} d \sigma=1 .
$$

Hence, $\left|G\left(a_{0}\right)\right|^{p} \geq 1$.

Since $G_{\beta}$ is analytic in $\overline{\mathbb{D}}$ and, by Theorem 3.2 , acts as an expansive multiplier on $A_{\alpha}^{p}$ for $0<\alpha \leq 1$, we have the following consequence of Theorem 4.2:

Corollary 4.3. $\left|G_{\beta}(z)\right| \geq 1$ for all $z$ in $\partial \mathbb{D}$.

\section{The main result.}

We now show that extremal functions in $A_{\alpha}^{p}$ can not have extra zeros in $\mathbb{D}$ when $0<\alpha \leq 1$ and $1 \leq p<\infty$.

Theorem 5.1. For $0<\alpha \leq 1$ and $1 \leq p<\infty$, let $G$ be an extremal function in $A_{\alpha}^{p}$ corresponding to an $A_{\alpha}^{p}$ zero-set. Then $G$ has no extraneous zeros in $\mathbb{D}$.

Proof. Suppose $G$ has an extra zero, $\beta$, for some $p$ and $\alpha$. Let $G_{\beta}$ be the corresponding single-point extremal function in $A_{1}^{p}$ given by Formula (2) and consider the function $G / G_{\beta}$. Since $\left|G_{\beta}(z)\right| \geq 1$ for all $z \in \partial \mathbb{D}$ by Corollary 4.3, it is not hard to see that $G / G_{\beta} \in A_{\alpha}^{p}$. Moreover,

$$
1=\|G\|_{p, \alpha}=\left\|G_{\beta}\left(G / G_{\beta}\right)\right\|_{p, \alpha} \geq\left\|G / G_{\beta}\right\|_{p, \alpha},
$$


by Theorem 3.2. Since the function

$$
\frac{G / G_{\beta}}{\left\|G / G_{\beta}\right\|_{p, \alpha}}
$$

is a competitor for the extremal property, we see that

$$
\operatorname{Re}\{G(0)\} \geq \operatorname{Re}\left\{\frac{G(0) / G_{\beta}(0)}{\left\|G / G_{\beta}\right\|_{p, \alpha}}\right\}>\operatorname{Re}\{G(0)\},
$$

where we have also used Lemma 2.1. From this contradiction, we see that $G$ can not have any extraneous zeros.

In particular, for $0<\alpha \leq 1$ and $1 \leq p<\infty$ the single-point extremal functions in $A_{\alpha}^{p}$ can not have any extra zeros in $\mathbb{D}$. Hence, these extremal functions must be given by Formula (2). Shimorin's results [11] allow us to conclude the same for $-1<\alpha<0$. We then have the following result about the corresponding hypergeometric functions:

Corollary 5.2. When $1 \leq p<\infty$ and $-1<\alpha \leq 1$, the hypergeometric function $F\left(-\alpha-1, \frac{p}{2} ; \frac{p}{2}+1 ; z\right)$ has no zeros in $\mathbb{D}$.

Using two well-known transformation formulas, we can prove additional corollaries involving hypergeometric functions. The first corollary is a consequence of Pfaff's transformation

$$
F(a, b ; c ; z)=(1-z)^{-a} F\left(a, c-b ; c ; \frac{z}{z-1}\right) .
$$

A derivation of this transformation can be found in [1].

Corollary 5.3. When $1 \leq p<\infty$ and $-1<\alpha \leq 1$, the hypergeometric functions $F\left(-\alpha-1,1 ; \frac{p}{2}+1 ; z\right)$ and $F\left(\frac{p}{2}+\alpha+2, \frac{p}{2} ; \frac{p}{2}+1 ; z\right)$ have no zeros in the half-plane $\operatorname{Re}\{z\}<\frac{1}{2}$.

By exploiting the symmetry of the hypergeometric function in the parameters $a$ and $b$, Pfaff's transformation can be used to establish Euler's formula

$$
F(a, b ; c ; z)=(1-z)^{c-a-b} F(c-a, c-b ; c ; z) .
$$

We can then conclude the following:

Corollary 5.4. When $1 \leq p<\infty$ and $-1<\alpha \leq 1$, the hypergeometric function $F\left(\frac{p}{2}+\alpha+2,1 ; \frac{p}{2}+1 ; z\right)$ has no zeros in $\mathbb{D}$. 


\section{Extremal functions for $0<p<1$.}

In [13], Formula (2) was established only when $1 \leq p<\infty$, since the proof required the use of certain Banach space results. We now show that when $\alpha=1$ the formula holds for $0<p<1$ also. This allows us to prove that, even for $0<p<1$, extremal functions in $A_{\alpha}^{p}$ can not have extra zeros when $0<\alpha \leq 1$.

For $0<p<1$, define the function $G_{\beta}$ as

$$
G_{\beta}(z)=\frac{\bar{\beta}}{|\beta|} \frac{\beta-z}{1-\bar{\beta} z}\left[\frac{F\left(-2, \frac{p}{2} ; \frac{p}{2}+1 ; \bar{\beta} \frac{\beta-z}{1-\bar{\beta} z}\right)^{2}}{F\left(-2, \frac{p}{2} ; \frac{p}{2}+1 ; 1\right) F\left(-2, \frac{p}{2} ; \frac{p}{2}+1 ;|\beta|^{2}\right)}\right]^{\frac{1}{p}} .
$$

In other words, let Formula (2) be the definition of $G_{\beta}$ when $0<p<1$ and $\alpha=1$. We first establish an orthogonality property of this function.

Lemma 6.1. For $0<p<1$, the function $G_{\beta}$ has the property

$$
\int_{\mathbb{D}}\left|G_{\beta}\right|^{p} f w_{1} d \sigma=f(0)
$$

for all $f \in h^{\infty}$.

Proof. First, using Equation (11), we can write $G_{\beta}$ in the form

$$
\frac{\bar{\beta} \varphi_{\beta}(z)}{|\beta|}\left[\frac{\left(1-|\beta|^{2}\right)^{3} F\left(\frac{p}{2}+3,1 ; \frac{p}{2}+1 ; \bar{\beta} \varphi_{\beta}(z)\right)^{2}}{2 B\left(\frac{p}{2}+1,2\right)(1-\bar{\beta} z)^{6} F\left(\frac{p}{2}+3,1 ; \frac{p}{2}+1 ;|\beta|^{2}\right)}\right]^{\frac{1}{p}},
$$

where $\varphi_{\beta}(z)=(\beta-z) /(1-\bar{\beta} z)$ and $B(x, y)=\Gamma(x) \Gamma(y) / \Gamma(x+y)$ is the Beta function. If $f$ is a polynomial, setting $\zeta=\varphi_{\beta}(z)$ we see that,

$$
\begin{aligned}
& \int_{\mathbb{D}}\left|G_{\beta}(z)\right|^{p} f(z) w_{1}(z) d \sigma(z) \\
& =\left[B\left(2, \frac{p}{2}+1\right) F\left(\frac{p}{2}+3,1 ; \frac{p}{2}+1 ;|\beta|^{2}\right)\right]^{-1} \\
& \quad \cdot \int_{\mathbb{D}}|\zeta|^{p}\left|F\left(\frac{p}{2}+3,1 ; \frac{p}{2}+1 ; \bar{\beta} \zeta\right)\right|^{2} f\left(\varphi_{\beta}(\zeta)\right)\left(1-|\zeta|^{2}\right) d \sigma(\zeta) .
\end{aligned}
$$

Letting $F\left(\frac{p}{2}+3,1 ; \frac{p}{2}+1 ; \bar{\beta} \zeta\right)=\sum_{n=0}^{\infty} b_{n} \zeta^{n}$ and

$$
F\left(\frac{p}{2}+3,1 ; \frac{p}{2}+1 ; \bar{\beta} \zeta\right) f\left(\varphi_{\beta}(\zeta)\right)=\sum_{n=0}^{\infty} c_{n} \zeta^{n},
$$


we find that

$$
\begin{aligned}
& \int_{\mathbb{D}}\left|G_{\beta}(z)\right|^{p} f(z) w_{1}(z) d \sigma(z) \\
& =\left[B\left(2, \frac{p}{2}+1\right) F\left(\frac{p}{2}+3,1 ; \frac{p}{2}+1 ;|\beta|^{2}\right)\right]^{-1} \\
& \cdot \sum_{n=0}^{\infty} B\left(2, \frac{p}{2}+n+1\right) \overline{b_{n}} c_{n} .
\end{aligned}
$$

Now, rewriting the definition of $F\left(\frac{p}{2}+3,1 ; \frac{p}{2}+1 ; z\right)$, we see that

$$
F\left(\frac{p}{2}+3,1 ; \frac{p}{2}+1 ; z\right)=\sum_{n=0}^{\infty} \frac{B\left(2, \frac{p}{2}+1\right)}{B\left(2, \frac{p}{2}+n+1\right)} z^{n},
$$

and so, $B\left(2, \frac{p}{2}+n+1\right) \overline{b_{n}}=B\left(2, \frac{p}{2}+1\right) \beta^{n}$. Thus,

$$
\begin{aligned}
\int_{\mathbb{D}}\left|G_{\beta}(z)\right|^{p} f(z) w_{1}(z) d \sigma(z) \\
=\left[B\left(2, \frac{p}{2}+1\right) F\left(\frac{p}{2}+3,1 ; \frac{p}{2}+1 ;|\beta|^{2}\right)\right]^{-1} \\
\quad \cdot \sum_{n=0}^{\infty} B\left(2, \frac{p}{2}+1\right) \beta^{n} c_{n} \\
=\left[F\left(\frac{p}{2}+3,1 ; \frac{p}{2}+1 ;|\beta|^{2}\right)\right]^{-1} \sum_{n=0}^{\infty} c_{n} \beta^{n}=f(0) .
\end{aligned}
$$

This shows that (13) holds for all polynomials $f$. It is clear that the result holds for harmonic polynomials also. Since $\left|G_{\beta}\right|^{p} \in L^{1}\left(\mathbb{D}, w_{1} d \sigma\right)$, we can conclude that the relation holds for all $f \in h^{\infty}$.

We note that (13) is equivalent to the condition

$$
\int_{\mathbb{D}}\left(\left|G_{\beta}\right|^{p}-1\right) f w_{1} d \sigma=0
$$

for all $f$ in $h^{\infty}$, using the mean-value property for harmonic functions.

Proposition 6.2. Suppose $0<p<1, \beta \in \mathbb{D} \backslash\{0\}$ and $\alpha=1$. Then the function $G_{\beta}$ defined by Formula (12) is the unique extremal function in $A_{1}^{p}$ corresponding to the zero-set $\{\beta\}$.

Proof. For $0<p<1$, the function defined by Formula (12) is analytic in $\overline{\mathbb{D}}$. Thus, the boundary-value problem $(7)$ has a solution $\phi \in C^{2}(\overline{\mathbb{D}})$ in this case also. In view of Lemma 6.1, we can then establish an analogue of Lemma 3.1 and use the representation for $\phi$ given in the second part of this lemma to show that $G_{\beta}$ acts as an expansive multiplier on $A_{\alpha}^{p}$ for $0<\alpha \leq 1$. Thus, by Theorem 4.2 , we see that $\left|G_{\beta}(z)\right| \geq 1$ for all $z \in \partial \mathbb{D}$. Hence, if $f \in A_{1}^{p}$ 
and $f(\beta)=0$, then $f / G_{\beta} \in A_{1}^{p}$ and $\left\|f / G_{\beta}\right\|_{p, \alpha} \leq\|f\|_{p, \alpha}$. We use this last property to show that $G_{\beta}$ is extremal.

Let $f \in A_{1}^{p}$ satisfy $f(\beta)=0$ and $\|f\|_{p, \alpha}=1$, and assume that $f$ is not a constant multiple of $G_{\beta}$. For every $g$ in $A_{\alpha}^{p}$, since $|g|^{p}$ is subharmonic, $|g(0)|^{p}<\|g\|_{p, \alpha}^{p}$, unless $g$ is constant. Thus since $G_{\beta}(0)>0$,

$$
\frac{\operatorname{Re}\{f(0)\}}{\operatorname{Re}\left\{G_{\beta}(0)\right\}} \leq\left|\frac{f(0)}{G_{\beta}(0)}\right|<\left\|f / G_{\beta}\right\|_{p, \alpha} \leq\|f\|_{p, \alpha}=1
$$

In other words, $\operatorname{Re}\{f(0)\}<\operatorname{Re}\left\{G_{\beta}(0)\right\}$.

Thus, the only remaining competitors for the extremal property are constant multiples of $G_{\beta}$. But since we know that the extremal function has norm 1 and is real and positive at the origin (see the remark after Lemma 2.1), $G_{\beta}$ is the only possible candidate.

We can now conclude that our main result holds for $0<p<1$ also.

Theorem 6.3. For $0<\alpha \leq 1$ and $0<p<1$, let $G$ be an extremal function in $A_{\alpha}^{p}$ corresponding to an $A_{\alpha}^{p}$ zero-set. Then $G$ has no extraneous zeros in $\mathbb{D}$.

\section{References}

[1] G.E. Andrews, R. Askey and R. Roy, Special Functions, Cambridge University Press, Cambridge, 1999, MR 2000g:33001, Zbl 0920.33001.

[2] P. Duren, D. Khavinson, H.S. Shapiro and C. Sundberg, Contractive zero-divisors in Bergman spaces, Pacific J. Math., 157 (1993), 37-56, MR 94c:30048, Zbl 0739.30029.

[3] _ Invariant subspaces in Bergman spaces and the biharmonic equation, Michigan Math. J., 41 (1994), 247-259, MR 95e:46030, Zbl 0833.46044.

[4] P. Duren and A. Schuster, Bergman Spaces, American Mathematical Society, Providence, RI, to appear.

[5] H. Hedenmalm, A factorization theorem for square area-integrable analytic functions, J. Reine Angew. Math., 422 (1991), 45-68, MR 93c:30053, Zbl 0734.30040.

[6] _ Open problems in the function theory of the Bergman space, Festschrift in honour of Lennart Carleson and Yngve Domar, (Uppsala, 1993), Acta Univ. Upsaliensis Skr. Uppsala Univ. C Organ. Hist., 58, Uppsala Univ., Uppsala, 1995, 153-169, MR 96j:46023, Zbl 0849.30039.

[7] H. Hedenmalm and K. Zhu, On the failure of optimal factorization for certain weighted Bergman spaces, Complex Variables Theory Appl., 19 (1992), 165-176, MR 95f:30064, Zbl 0768.30006.

[8] K.Y. Osipenko and M.I. Stessin, On optimal recovery of a holomorphic function in the unit ball of $\mathbb{C}^{n}$, Constr. Approx., 8 (1992), 141-159, MR 93d:32008, Zbl 0757.41035.

[9] S.M. Shimorin, Factorization of analytic functions in weighted Bergman spaces, Algebra i Analiz, 5(5) (1993), 155-177 (Russian); St. Petersburg Math. J., 5 (1994), 1005-1022, MR 95j:30032, Zbl 0833.30031. 
[10] _ On a family of conformally invariant operators, Algebra i Analiz, 7(2) (1995), 133-158 (Russian); St. Petersburg Math. J., 7 (1996), 287-306, MR 96i:47010, Zbl 0855.30040.

[11] The Green function for the weighted biharmonic operator $\Delta\left(1-|z|^{2}\right)^{-\alpha} \Delta$, and factorization of analytic functions, Zap. Nauchn. Sem. S.-Peterburg. Otdel. Mat. Inst. Steklov. (POMI), 222 (1995), 203-221 (Russian); J. Math. Sci., 87(5) (1997), 3912-3924., MR 97c:31005, Zbl 0896.31001, Zbl 0909.31004.

[12] D. Vukotić, private communication.

[13] R.J. Weir, Canonical divisors in weighted Bergman spaces, Proc. Amer. Math. Soc., 130(3) (2002), 707-713 (electronic), CMP 1866 024, Zbl 0988.32001.

Received October 23, 2001.

Department of Mathematics

UNIVERSITY OF VIRGINIA

Charlottesville, VA 22904

E-mail address: rw8t@virginia.edu 